\title{
Evaluation of Progressive Treemaps to Convey Tree and Node Properties
}

\author{
René Rosenbaum and Bernd Hamann \\ Institute for Data Analysis and Visualization (IDAV), Department of Computer Science, \\ University of California, Davis, Davis, CA 95616-8562, U.S.A.
}

\begin{abstract}
In this paper we evaluate progressive treemaps. Progressive refinement has a long tradition in image communication, but is a relatively new approach for information presentation. Besides technical benefits it also promises to provide advantages important for the conveyance of data properties. In this first user study in this domain, we focus on the additional value of progressive refinement for traditional treemaps to convey the topology of a given hierarchical data set and properties of its nodes. To achieve this, we compare the results gained for common squarified treemap displays with and without progression for various related tasks and set-ups. The results we obtained indicate that progressive treemaps allow for a better conveyance of topological features and node properties in most set-ups. We also assessed the opinions of our study participants and found that progressive treemaps also lead to a better confidence about the given answers and provide more assistance and user friendliness.
\end{abstract}

Keywords: Progressive refinement, Treemap display, Data presentation, Usability, User tests.

\section{INTRODUCTION}

Long response times and visual clutter caused by the need to process and display large volumes of data are major problems in visualization. They result in an undesirable viewing experience and unreadable information displays. In raster image communication those problems have been overcome by the development of progressive refinement schemes. Despite the various benefits of the approach, progressive information displays have just recently been proposed. One example are progressive treemaps ${ }^{1}$ (PTM) enhancing common treemap displays (TM) by progression (see Figure 1). Traditional treemaps have been broadly accepted by end users to represent hierarchical data, often referred to as trees. However, if the amount of data increases the tree structure and properties, especially of inner nodes, cannot easily be conveyed. ${ }^{2}$ It was demonstrated that the many previews and the geometry refinement provided by progressive treemaps can solve the problems arising from large data sets, but qualitative statements made earlier have never been evaluated.

In this publication, we present the results obtained from a user study evaluating the benefits of PTMs for conveying tree topology and node properties. It is the first study in this domain and based on the comparison of a static and a progressive presentation strategy both using a squarified treemap layout in a variety of test set-ups. We provide quantitative evidence that viewers of PTMs have a better understanding of the tree and its nodes, are more confident about their answers, and have a more pleasant viewing experience than viewers using TMs. This observation applies to large as well as small form-factor screens making PTMs a more appropriate means for a broad variety of information displays. Thus, PTM are able to convey data characteristics for which a TM fails leading to a more complete visualization technique and broader adoption of treemaps by the users.

Section 2 reviews the TM approach and its various extensions as well as PTMs and their technical and semantical benefits related to this evaluation. We describe the focus of our study in Section 3 and its design and methodology in Section 4. The obtained results are presented in Section 5 and used in Section 6 to answer the research questions we are focussing on. Conclusions and directions for future research are provided in Section 7.

Further author information: (Send correspondence to René Rosenbaum)

René Rosenbaum: E-mail: rosenbaum@ieee.org

Bernd Hamann: E-mail: hamann@cs.ucdavis.edu 


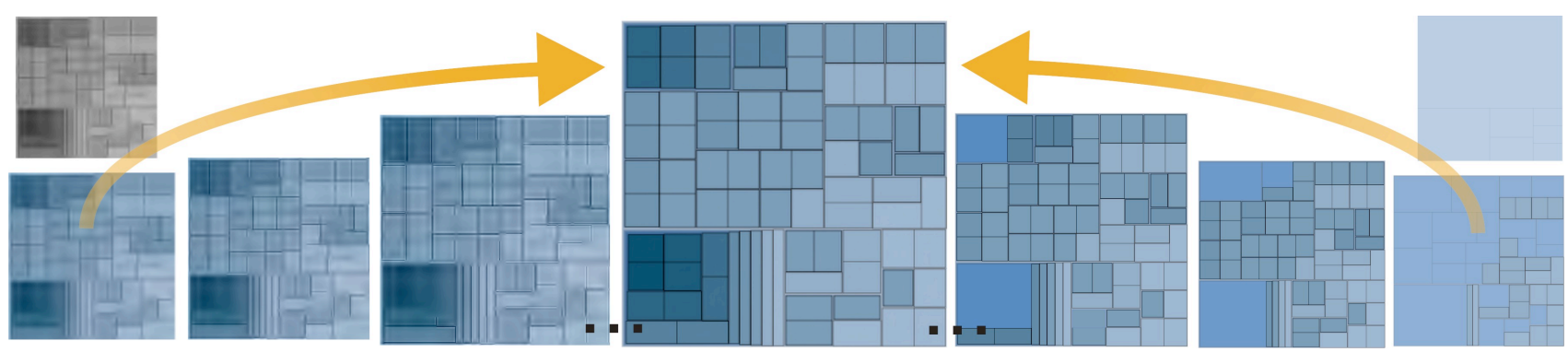

Figure 1. Traditional pixel-based progressive refinement of a rendered treemap display (left) and the used refinement approach based on the underlying geometry (right). The final representation (center) is the same in either case.

\section{TREEMAPS, PROGRESSIVE REFINEMENT, AND RELATED WORK}

The treemap display belongs to the class of hierarchy visualizations implicitly communicating parent-child relationships. This is achieved by representing the tree as recursively nested rectangular cells (see Figure 1, center). This containment approach illustrates the structure of the tree and clearly identifies parent and child nodes belonging to the same subtree. Size, color, and other graphical cell attributes thereby can be used to represent additional data characteristics.

Using all the available space treemaps have been proven to convey well the characteristics of the leaf nodes making them useful for small screen displays and application domains where leaf nodes are of main interest. However, a treemap has three distinct drawbacks: (1) tree topology is not well conveyed by the nesting property, ${ }^{2}$ (2) inner hierarchy nodes are occluded, ${ }^{2}$ and (3) the rectangles associated with nodes become small in size and indiscernible for large hierarchies.

To overcome these disadvantages, the treemap display has been steadily improved. Thereby, existing publications focus on layout and appearance. The traditional slice-and-dice approach ${ }^{3}$ was enhanced by squarified, ${ }^{4}$ cushion, ${ }^{5}$ Voronoi, ${ }^{6}$ or generalized layouts. ${ }^{7}$ There are also radial layouts ${ }^{8}$ and different three-dimensional approaches. ${ }^{9}$ Cascaded treemaps ${ }^{10}$ have been developed to emphasize containment within tree structure, but at the cost of space. There are strategies to emphasize important nodes by applying spatial distortion. ${ }^{11}$ However, even when these enhancements are applied, the inherent property to draw child nodes on top of parent nodes prevents a general solution for the fundamental problems. It was shown ${ }^{12}$ that suitable means for interaction ${ }^{13}$ lead to a much better understanding of the tree, but this requires time and knowledge in data exploration.

Progressive treemaps aim at increasing the (1) efficiency of data storage and communication and (2) the conveyance of knowledge about the tree and its nodes. Based on the principle of pixel-based progressive refinement widely used in modern image communication to transmit photos efficiently (see Figure 1, left), a PTM incrementally refines the geometry of the treemap. Refinement starts with the rectangle associated with the root node and usually continues tree level-wise until all nodes are displayed (see Figure 1, right). The idea is that the first tree levels can be provided to the user with little data leading to an instantaneous system response. Inherent scalable compression and means to constrain the transmission by the geometry-of-interest concept lead to a highly resource-efficient data communication. ${ }^{1}$

The PTM approach is widely applicable and can be applied together with almost all of the mentioned treemap extensions. The display can be refined uniformly or by author-based or interactive geometry-of-interest. This adds further benefits useful for narrative and interactive visualization systems, ${ }^{1,14}$ such as the one described in $\left.{ }^{13}\right]$. Due to their scalable manner, PTMs are also well-suited for the adaptation to the respective viewing device. $^{15}$

There is no approach without shortcomings. The main problem of PTMs concerns the handling of dynamic data. ${ }^{14}$ In case nodes or their values change, the whole hierarchy must be re-processed and -compressed as well as refinement restarted. This, however, is a general problem when data is stored or transferred in compressed representation. Furthermore, the advantages of PTMs are strongly constrained when the tree has only a few hierarchy levels. 
Although the technical benefits of PTMs are the most obvious reasons for their application, it was claimed that they are also able to enhance the conveyance of properties of the data. ${ }^{1,14}$ Depending on the application domain, this can lead to faster data browsing, node search, or higher accuracy of task completion. These claims stem from their following inherent characteristics:

Multiple views of the data Providing multiple views of the data has been proven to perform well in most areas of visualization. This property is supported by the different previews shown during the refinement of the treemap. When the treemap is refined, each preview displays the inner nodes of the respective hierarchy level without occlusion. This has the significant advantage that all these nodes can be thoroughly examined. Contrary to the common multiple window systems, progression provides these previews in a single window making it a better fit for devices with constrained screen space.

Incremental detail refinement Providing the data at multiple detail levels is another widely accepted concept in visualization. PTMs inherently support this concept by incremental refinement. This promises to lead to a successive addition of knowledge about the data and in turn to a better mental model of the data.

Data high-lighting Visual changes between the previews caused by the addition of new node rectangles intuitively attract the attention of the viewer to the associated regions within the treemap.

Information-seeking mantra ${ }^{\mathbf{1 6}}$ The mantra calls for a better understanding of the data by providing them at multiple abstraction levels starting from an overview and proceeding to desired details. PTMs support this mantra by their provided means for detail-scalable representation and importance-driven refinement by geometry-of-interest.

Contrary to evaluations showing the value and appropriateness of the TM approach, ${ }^{2,12,17-20}$ no evaluations of PTMs have been published to date.

\section{FOCUS OF OUR EVALUATION}

Traditional treemaps are not able to properly convey tree topology and characteristics of inner nodes preventing this popular information display from being even more broadly applied. Progressive treemaps provide benefits that promise to overcome these drawbacks, but this has never been empirically evaluated. The presented user study represents an effort to fill this gap and was designed to answer the following questions:

1. Do progressive treemaps achieve a better conveyance of the tree topology to the viewer?

2. Do progressive treemaps achieve a better conveyance of properties of tree nodes to the viewer?

3. Do progressive treemaps lead to a more pleasant user experience?

Focussing on accuracy of task completion, we found profound answers to those questions in our study. Interaction has already been proven to increase the performance of TMs and has not been part of the study.

\section{DESIGN AND METHODOLOGY}

\subsection{Testing environment}

We used the Amazon Mechanical Turk micro-task service as a platform, which has been proven to perform well concerning user tests. ${ }^{21}$ After accepting a task page, participants were required to answer a web-based questionnaire. The results were reviewed in order to reject improper work.

\subsection{Participants}

In order to allow for a high quality user study, we only accepted participants that had a high reputation within the system (task approval rate above 95\%). Potential participants also had to finish a qualification test describing the principles of treemaps and requiring them to answer sample questions. 41 distinct participants where recruited for the user study. The majority had basic $(45 \%)$ or advanced $(37 \%)$ knowledge in data visualization, but never used treemaps. The remaining users had either no (11\%) or expert knowledge in data visualization (7\%). 


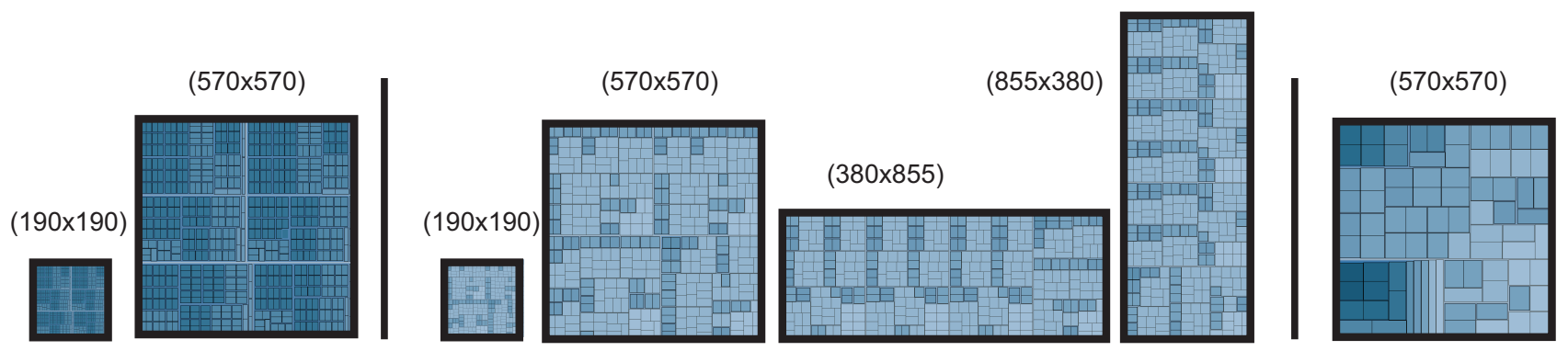

Figure 2. The different data sets, display layouts, and the resulting treemap representations used in the study: $D S 1$ (left), $D S 2$ (center), and $D S 3$ (right).

\subsection{Visual stimuli}

From the many different variations of treemaps that might be used to demonstrate the abilities and benefits of PTMs, we focussed on squarified treemaps. This layout is broadly applied and more sophisticated than the initial layout. As color is often applied to encode additional properties of the data, it was also used; in our case to indicate the depth of a node in the tree. The darker the color the deeper the node.

In order to test whether different spatial dimensions influence the test result, we provided treemaps in different sizes and page layouts (see Figure 2). We used a small (190x190) and a larger (570x570) display to show the data. Both displays are sufficiently small to impose some of the limitations of treemaps, without requiring larger data sets that might not be appropriate for meaningful processing by the hardware of the participants. This was necessary as the mechanical turk environment does not allow one to specify the test hardware. We also tested treemaps in landscape $(380 \mathrm{x} 855 \mathrm{px})$ and portrait layout $(855 \mathrm{x} 380 \mathrm{px})$ to allow to assess possible variances in the test results.

The applied treemap algorithm, the visual encodings, and set-ups were identical for TM and PTM.

PTMs were refined uniformly with one tree level per preview. A constant preview time of 1.5 seconds was used to avoid results distorted by a varying bandwidth in the uncontrolled mechanical turk environment. After a treemap had been fully refined, we provided options to replay the refinement similar to a reload button in a browser.

\subsection{Test set-up}

We created a test series that compared the performance of TMs and PTMs for various configurations and consisted of individual tests. Each test was shown on an individual page. Each participant was allowed to perform all or individual tests from the series. Each test page had a similar structure. It consisted of 4 parts: (1) user expertise, (2) TM, (3) PTM, and (4) user opinion. We limited the time to finish a test page to 10 minutes.

Part 1 asked for the current user expertise. One question ( What is your expertise in data visualization?") to the respective knowledge in visualization was asked to better understand the expertise of the audience. 4 answers ranging from expert to no knowledge were provided.

Parts 2 and 3 were nearly identical. The respective visualization - a TM or PTM — was shown at the top of the paragraph followed by 5 test questions (see Tasks) and one final question asking for the confidence about the given answers ("What is your confidence about the given answers?"). Part 2 showed a static image with a TM representation of a data set, part 3 a dynamic Java applet with a PTM, both visualizing a different data set. The participants were asked to examine each visualization independently. To state confidence, 5 answers on a Likert-scale were provided.

Part 4 was dedicated to receive general user feedback. Although users may not always like what works best for them, their subjective evaluation is an important part of a comparison. ${ }^{2,18-20}$ We were interested in their opinion concerning assistance ( "Which display assisted you most in decision making?", 2 answers: TM, PTM) and fun ("Which display did you enjoy most?", 2 answers: TM, PTM) while accomplishing the tasks for either kind of display. 
We used three artificial data sets in our tests: DS1 (9 level, 1066 nodes, balanced), DS2 (7 level, 511 nodes, medium-balanced), and $D S 3$ (12 level, 146 nodes, unbalanced). These data sets were created in accordance to the focus of our study. The number of nodes corresponds to the small display dimensions. The different set-ups and resulting visual appearance for all nodes are shown in Figure 2. For the remainder of the paper, an individual set-up is labeled $D S$ a-b-c, whereby $a$ refers to the id of the data set $(a \in\{1,2,3\}), b$ to the display approach $(b \in\{T, P\} ; T:$ TM, $P: \mathrm{PTM})$, and $c$ to the respective spatial layout $(c \in\{\emptyset, s, P, L\} ; \emptyset$ : large, $s$ : small, $P$ : portrait, $L$ : landscape). These set-ups allow one to test the influence of many different data and display properties and thus enable the broad applicability of the study.

\subsection{Tasks and hypotheses}

The tasks the participants had to accomplish were stated by 5 different self-explaining questions. All tasks corresponded to the benefits that PTM promised to provide to overcome the drawbacks of TMs (cf. to Section 2). The questions were identified in prior work and a pre-study testing 15 users in a broad variety of treemap-related tasks. The ones chosen for this user study were the ones that indicated most potential for PTMs. The particular questions and our hypotheses about the resulting outcomes are summarized in the following:

Q1 - "How many levels is the tree deep?" (Topology)

Answers: 5 answers, ordinal scale

This question was also of interest in $\left[^{2}\right]$ and refers to the ability of progression to provide multiple views of the data. As a level-wise refined PTM provides a preview for each single tree level, their number clearly indicates the depth of the tree. This promised to convey the depth of the tree much better than by containment alone. Hypothesis - H1: A PTM out-performs a TM in terms of the conveyance of the number of tree levels.

Q2 - "Of how many nodes is the tree comprised?" (Topology)

Answers: 5 answers, ordinal scale This question also refers to the multiple views of the data provided by progression, but also to the additional incremental refinement of the data. A PTM provides an uncluttered view to the inner nodes simplifying the estimation of their number for each tree level. Adding these estimations leads to the total number of nodes. Compared to the display of all nodes, this promised to make possible a better estimation of their number.

Hypothesis - H2: A PTM out-performs a TM in terms of the conveyance of the overall number of tree nodes.

\section{Q3 - "Is there a variation in the depth of the tree nodes?" (Topology)}

Answers: 4 answers, Likert scale

This questions was also of interest in $\left.{ }^{2,12}\right]$ and refers to the ability of progression for inherent data high-lighting. During progression only nodes that have children are refined. In a balanced tree this leads to a uniform refinement. Refining an unbalanced tree leads to scattered and local refinement. This can be easily observed and promised to convey this node property with higher accuracy.

Hypothesis - H3: A PTM out-performs a TM in terms of the conveyance of the balancing characteristic of the tree.

Q4 - "Where in the first tree level is the node with the highest number of sub-nodes located?" (Node properties)

Answers: 10 categorical answers related to the spatial position of a node

This task required the participants to compare inner nodes and has been of broad interest in related studies. ${ }^{12,18,19}$ In our study, it refers to the property of progression to provide multiple views of the data. The different previews show the inner nodes uncluttered. Thus, nodes residing at the first tree level can be much better identified and compared within a PTM than by a TM showing all nodes at once.

Hypothesis - H4: The position of the node in the first hierarchy level with the largest associated subtree is better determined when a PTM instead of a TM is used. 
Q5 - "Where is the deepest node located?" (Node properties)

Answers: 10 categorical answers related to the spatial position of a node

This task refers to the ability of PTMs for data high-lighting and is of interest for TMs in $\left[{ }^{12,19}\right]$. The question is similar to Q3 but refers to a single node instead of the whole tree. The node that is refined last represents the deepest node promising to make possible its much simpler identification as by color alone.

Hypothesis - H5: The position of the deepest tree node is better determined when a PTM instead of a TM is used.

In our user study we did not test the support of PTMs for the information seeking mantra. This would have required a geometry-of-interest set-up which was beyond the scope of this first study.

The benefits of PTMs also suggested to test the following two hypotheses related to user experience:

Hypothesis - H6: A PTM leads to more confidence about the gained knowledge compared to a TM.

Hypothesis - H7: A PTM provides more assistance and fun compared to a TM.

\section{RESULTS}

The answers of the participants were analyzed statistically (One-way ANOVA). The confidence interval was set at $95 \%$ for all analysis. We cleaned the test data from extreme outliers indicating a misuse of our test platform. These outliers were found by comparing the test results of an individual participant to the average results. If the results significantly differed in at least four out of the five questions, we removed the whole test record of the participant. When not stated otherwise, the results obtained from the multiple set-ups were pair-wise highly significantly different from each other $(p<0.01)$. The same applied to the presented means. We discuss the obtained results for objective ("Correctness") and subjective questions ("Confidence" and "User preferences") separately.

\begin{tabular}{|c|c|c|c|c|c|c|c|c|c|}
\hline Question & \multicolumn{2}{|c|}{ Average (\%) } & \multicolumn{2}{c|}{ Standard deviation (\%) } & \multicolumn{2}{c|}{ Best $(\%)$} & \multicolumn{2}{c|}{ Worst (\%) } & Average gain of \\
& trad. & prog. & trad. & prog. & trad. & prog. & trad. & prog. & progression (\%) \\
\hline Q1 & 27.7 & 49.8 & 10.1 & 9.8 & 45.8 & 62.1 & 17.9 & 39.3 & 22.1 \\
\hline Q2 & 47.6 & 55.8 & 21.0 & 23.8 & 69.0 & 79.2 & 17.9 & 19.6 & 8.2 \\
\hline Q3 & 44.2 & 52.0 & 16.8 & 12.5 & 62.5 & 64.3 & 14.3 & 28.6 & 7.8 \\
\hline Q4 & 31.5 & 43.5 & 8.7 & 11.9 & 44.8 & 57.1 & 21.4 & 32.1 & 12.0 \\
\hline Q5 & 40.5 & 45.8 & 22.3 & 22.65 & 71.4 & 75.0 & 14.3 & 20.7 & 5.3 \\
\hline
\end{tabular}

Table 1. Statistical summary for the five tasks and test questions.

\subsection{Correctness}

The following discussion with regard to the correctness of the answers mainly focusses on the differences in the results obtained for TM and PTM and neglects statements regarding general properties of treemaps in the evaluated settings. A statistical summary and the absolute correctness obtained for either kind of display are shown in Table 1. After providing an overview about the general test results for each task, we discuss the details with regard to the influence of the individual properties of the data and viewing parameters used in the tests.

\section{Q1 - Number of tree levels}

We found that PTMs performed always better than TMs (see Figure 3, left). This statement holds for all individual data sets and display configurations. Compared to other tasks the highest gain of PTMs compared to TMs was obtained. PTMs performed on average $22.1 \%$ better than TMs. This finding shows the strong influence and support of the tree level-wise refinement and incremental presentation provided by progressive refinement. The standard deviation was almost identical for both displays. 
Best performance and accuracy for both kinds of display was achieved for data set $D S 2$. We explain this by the fact that the number of levels and thus the efforts for the viewer for their determination were least. The differences were highest for the data set with many nodes $(D S 1, D S 2)$ and in small representation $(D S 1)$. A treemap showing many or very small nodes is so cluttered that levels cannot be recognized suitably. This is emphasized by the results obtained for data set $D S 1$ displayed by a TM which were not significantly different from picking an answer randomly. The smallest difference was obtained for data set $D S 3$. The rectangles of the treemap are much better discernible with fewer nodes and a larger screen in turn leading to less influence of progressive refinement.

Nodes: The number of nodes is the main reason why the traditional approach fails for $D S 1$. Except for $D S 2$ the results obtained for other data sets are similar leading to the conclusion that the depth of the tree is difficult to convey with TMs.

Balanced: In our experiments, we found no evidence that the balancing property of the tree influences the correctness of the answers to this question.

Levels: The data set with the least levels performed best for progression. For a larger number of levels correctness was almost identical. We explain this by the fact that users might make errors or simply stop counting when the number of levels becomes too high.

Display size: We found that $D S 1$ displayed on a small screen performed better when progressively refined rather than on the large screen. We found no explanation for this phenomenon which was not repeated for $D S 2$. The difference between TM and PTM in this set-up was also higher.

Layout: Although we obtained the best results with the square layout, the layout did not have a strong influence to accomplishing the task successfully. Most differences between layouts were found by using the traditional approach.

Hypothesis: The results we obtained strongly support hypothesis H1.
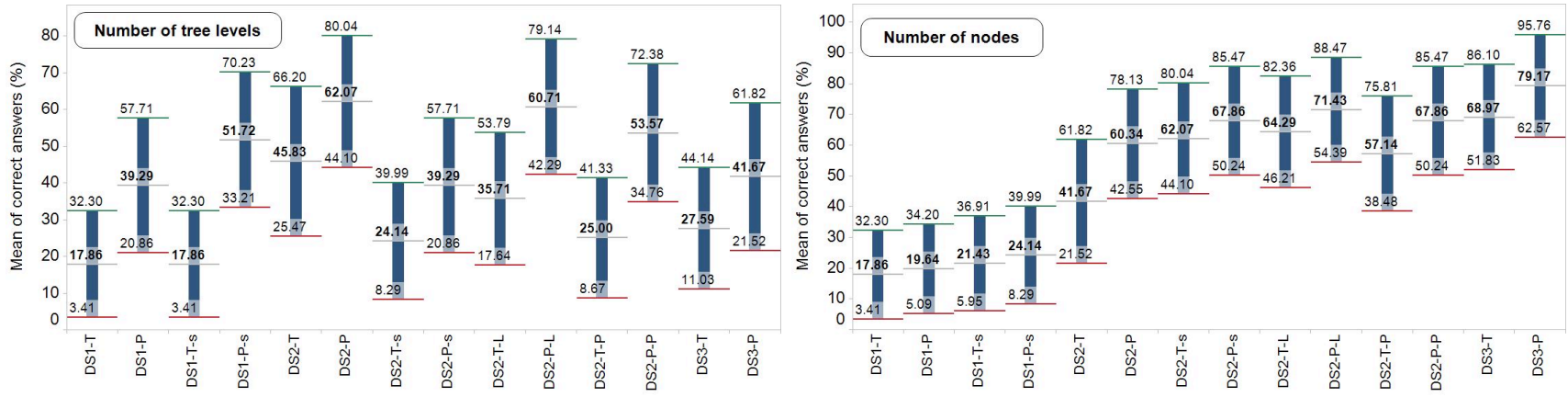

Figure 3. Correct answers we obtained for the provided questions concerning the number of tree levels (left) and the number of nodes (right). The green/upper and red/lower marks in the charts indicate the respective $95 \%$ confidence interval.

\section{Q2 - Number of nodes}

PTMs perform for all set-ups better than TMs and showed the highest rate of correct answers of all tasks (see Figure 3, right). The average gain of PTMs, however, was much less than for the tree level task $(8.2 \%)$. The standard deviation was very high and similar for both display strategies. The highest gain of approximately $19 \%$ was obtained for data set $D S 2$, the highest accuracy for data set $D S 3$ having the least nodes of all data sets. The answers we obtained for data set $D S 1$ in both set-ups and $D S 2$-T were statistically not different from a random selection. 
Nodes: The more nodes had to be displayed, the smaller was the number of correct answers. When the number of nodes becomes too large, the display becomes cluttered and it is more difficult to estimate their correct number. This has already been shown for Q1 and also applies for PTMs. In such cases, the benefit of additional views does not allow one to increase the number of correct answers significantly (DS1-P) or strongly (DS1-P-s). Interestingly, the absolute rate of correctness does not change strongly when sufficiently less nodes are displayed ( $D S 2, D S 3)$.

Balanced: We found no evidence that the balancing property of tree levels influences the correctness of the answers to this question.

Levels: In our experiments, we found no evidence that the number of tree levels influences the correctness of the answers to this question.

Display size: No strong differences in the gain for both kinds of displays and display sizes were found.

This is interesting as we expected that smaller displays would benefit more from progressive refinement. For the significant results we obtained for $D S 2$ we encountered the same phenomenon as observed during the analysis of the answers given for Q1 - small treemaps showed a higher rate of correct answers than large treemaps Layout: The layout does not strongly influence the number of correct answers. Highest variations were obtained for TMs.

Hypothesis: The results we obtained support hypothesis H2.


Figure 4. Median and 95\%-confidence intervals of correct answers we obtained for the provided questions concerning the balancing of the tree (left) and the position of the largest subtree (right).

\section{Q3 - Balancing}

PTMs performed always better or identical to TMs (see Figure 4, left). The average gain of PTMs was comparable to Q2 (7.8\%). We explain the generally large number of correct answers for both kinds of display mostly by the fact that the color coding of the nodes also conveys the balancing characteristic - uniform color of the treemap represents a balanced tree, a textured treemap an unbalanced tree. A high standard deviation was obtained for TM. Most test pairs showed an identical pattern in the difference. The differences varied between 7.2 and $15.7 \%$. The largest gain for PTMs was obtained for $D S 1$ displayed on a small display. There was no significant difference in the two strategies for $D S 2$ and $D S 3$ when shown on a large display. $D S 2$ displayed by a TM in portrait mode was not significantly different from a random answer.

Nodes: In our experiments, we found no evidence that the number of nodes influences the correctness of the answers to this question.

Balanced: The balancing of the tree has the most influence on the performance gain of PTMs. However, it only provides an advantage when the tree is balanced.

Levels: In our experiments, we found no evidence that the number of tree levels influences the correctness of the answers to this question.

Display size: We found that PTMs performed much better on small display sizes. Interestingly, this also applies to the medium-balanced data set $D S 2$ that did not show a significant gain of PTMs on the large display. 
Layout: Interestingly, the layout also had a strong influence on correctness. Although best results were obtained by the square layout, the difference between TM and PTM was much larger when the screen had a certain orientation. These differences were significant and between 7.2 and $14.3 \%$. The highest difference was obtained in portrait mode, but we relate this to the week performance of TMs in this specific set-up.

Hypothesis: The results we obtained support hypothesis H3.

\section{Q4 - Position of largest subtree}

Our results showed that PTMs performed always better for this comparison task than TMs (see Figure 4, right). Thereby, progressive refinement leads to an average gain of $12 \%$ compared to the static presentation. However, the standard deviation was higher for PTMs. The different gains were similar for all test set-ups and ranged between 7.1 and $19.4 \%$. The highest correctness and gain was obtained for $D S 2$ displayed in square layout. The smallest gain was obtained for $D S 1$.

Nodes: In our experiments, we found no evidence that the number of nodes influenced the correctness of the answers to this question. Lowest correctness was obtained for $D S 1$ having the most number of nodes for TM as well as PTM. PTMs, however, strongly reduce the number of nodes in the relevant level leading to our conclusion.

Balanced: Balancing has only a minor influence on correctness. Considering data sets $D S 2$ and DS3 there was only a marginal difference in the number of correct answers. Both, TM and PTM, led to a high level of correctness. The low number of correct answers obtained for data set $D S 1$ stems from the fact that the balancing property led to many nodes of almost identical size. We expected PTMs to achieve a higher gain, but suspect that this was prevented by the short display time for each preview.

Levels: In our experiments, we found no evidence that the number of tree levels influenced the correctness of the answers to this question.

Display size: Surprisingly, the results did not differ strongly for the different screen sizes. We explain this by the fact that in the second preview rectangles were still large enough to be meaningfully compared even on the small screen. For data set $D S 2$, the performance on the small display was even slightly better.

Layout: Layouts different from the square layout seemed to have confused the participants. This applies to both displays. Correctness obtained for both orientations was even less than that obtained for the small display. This was unexpected. Further investigation revealed that this was due to the fact that in both orientations the correct

node was overlapping the center, probably leading to a confusion of the participants about the correct answer. Hypothesis: The results we obtained strongly support hypothesis H4.

\section{Q5 - Position of deepest node}

PTM performed slightly better for this task than TMs (cf. Figure 5, left). The differences between both displays were the smallest for all tasks. The average gain was $5.3 \%$. We explain this by the fact that the depth of a node was also encoded by the color of the nodes. Standard deviation was high and almost identical in both cases. Best overall correctness was obtained for data set $D S 3$. This is not surprising, as the effect of color coding to answer the question is most effective in unbalanced trees. Contrary, the highest gain of PTMs (14.3\%) was obtained for $D S 1$ indicating that its means for data high-lighting are beneficial for such problematic data sources. We were not able to obtain a significant difference in the correctness of the answers for the presentation of $D S 1$ and $D S 2$ on small screens. Overall correctness in these set-ups was generally very low.

Nodes: We found that the more nodes were displayed, the smaller the number of correct answers. This mainly stems from the fact that with more nodes the associated rectangles become smaller and a larger number of colors (TM, PTM) or visual discontinuities (PTM) must be checked and observed.

Balanced: The balancing property also has an impact on the correctness of the answers. The more balanced the tree is, the more difficult it is to find the correct node. This applies to both displays. Balanced trees lead to almost similar colors of the tree nodes. The data high-lighting provided by PTMs does not help to overcome this issue as it leads to many and widely scattered visual changes. Although all provided example data sets had a single deepest node, the results indicate that it was difficult to notice. 
Levels: In our experiments, we found no evidence that the number of tree levels influenced the correctness of the answers to this question.

Display size: Display size also influenced correctness as well as the gain of PTMs. When the nodes become too small, differences in color or visual updates are not noticeable anymore. This led to almost identical results for both kinds of displays in the small representation.

Layout: We obtained almost identical results for treemaps displayed in square and portrait layout. After further analysis, we found that the exceptional performance of the landscape mode was related to the fact that in this layout the deepest node had a great exposure and thus was easy to spot and recognize.

Hypothesis: The results we obtained support hypothesis H5.
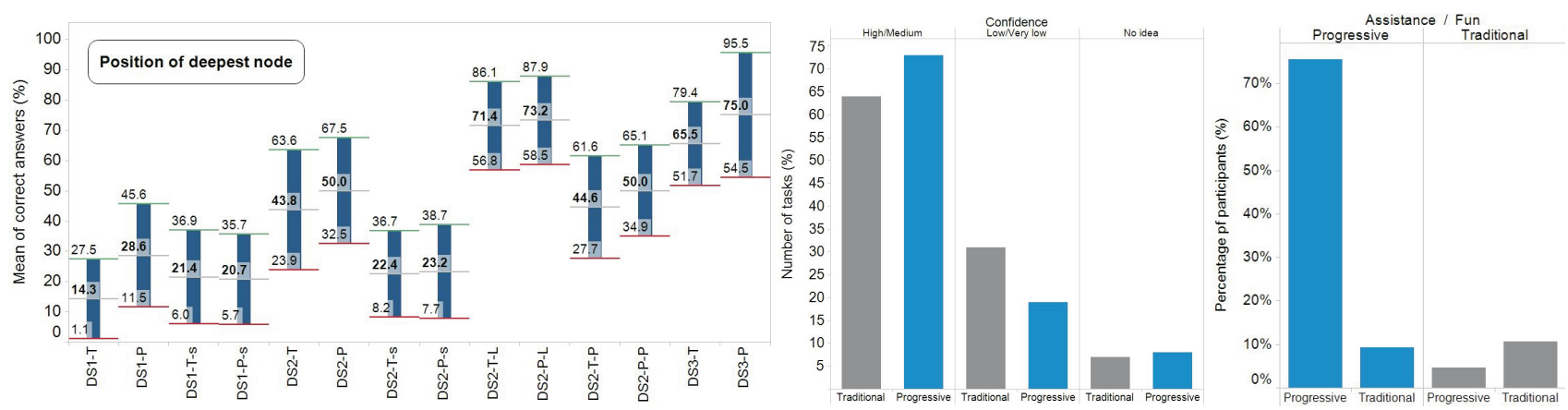

Figure 5. Median and 95\%-confidence intervals for answers regarding the position of the deepest node (left). The results obtained from questions related to confidence in the given answers (center) and stated user preferences (right) indicate that progressive treemaps provide more assurance, assistance, and fun.

\subsection{Confidence for given answers}

We found that $73 \%$ of all participants were confident with their answers when using a PTM contrary to $64 \%$ when using a TM (see Figure 5, center). $19 \%$ in case of the PTM and $31 \%$ in case of the TM were not confident in their answers. The percentage of users that were unsure about their answers was slightly higher for PTM (8\%) than for TM (5\%), which might be due to the novelty of the approach. These results support hypothesis H6.

\subsection{User preferences}

As shown in Figure 5, right, there was a strong response for the progressive approach when participants were asked for assistance and fun. $71 \%$ of all users voted for progression in both aspects. The majority of the participants (82\%) stated that PTMs assisted them better in accomplishing the task than TMs. $76 \%$ had more fun when using a progressive treemap. These results indicate that there is a strong support for hypothesis H7.

\section{REASONING}

The results obtained support the hypotheses of the study. We also verified that treemaps have problems conveying tree topology and properties of inner nodes. We now formulate the answers to the initial research questions.

Do progressive treemaps achieve a better conveyance of the tree topology to the viewer? The strong support of hypothesis $\mathbf{H 1}$ and support for hypotheses $\mathbf{H 2}$ and $\mathbf{H 3}$ indicate that progressive treemaps achieve a better conveyance of the tree topology to the viewer. The multiple views of the data, the incremental detail refinement, and inherent data high-lighting are additional means to convey the structure of the tree and lead to significant improvements compared to a static TM. Our data indicates that the degree of advancement depends on the properties of the data and applied viewing parameters. While the number of nodes, tree balancing, number of levels, and variations in screen size usually led to the expected results, there was no clear finding for the different layouts. Both orientations seemed to have confused the participants as they usually lead to worse results than obtained for the large square layout. Our findings also indicate that progressive refinement has limitations and does not always deliver performance gains. In our experiments, the obtained gain decreased for 
Q1 with an increase in the number of tree levels, for Q2 with an increase in the number of nodes, and for Q3 when the tree was not balanced.

Do progressive treemaps achieve a better conveyance of properties of tree nodes to the viewer? The multiple views provided by PTMs are the main reason for the strong support of H4. All the inner nodes that correspond to the current refinement stage are displayed without occlusion and often without clutter making possible a much better comparison than with TMs. Although the deepest node is also fully visible in a TM, it was shown that the inherent means of PTMs for data high-lighting lead to higher correctness of the answers, supporting H5. Only the deepest node is added to the display at the end of the refinement intuitively drawing attention to this node. Both findings indicate that progressive treemaps achieve a better conveyance of properties of tree nodes to the viewer. Similar to the results obtained for tree topology, the achieved gain depends on the used data and viewing parameters. Although, PTMs usually led to a significantly higher number of correct answers, we revealed the limitation that that very short update times do not allow for a sufficient visual analysis of inner nodes (Q4). Further, when nodes become so small, differences in the visual updates are not noticeable (Q5). Both cases lead to a strong decrease in the gain that progressive refinement can provide.

Do progressive treemaps lead to a more pleasant user experience? Participants were more confident when using a PTM rather than a TM, supporting H6. Furthermore, the vast majority of participants preferred PTMs with regard to assistance and fun, strongly supporting $\mathbf{H 7}$. We assume that this is mainly due to the many additional views and an incremental build-up of insight leading to a much better understanding of the tree, its different levels, relationships, and nodes. The property that most of the views are uncluttered also contribute to the success. We see the support of $\mathbf{H 6}$ and $\mathbf{H 7}$ as strong indicators that progressive treemaps lead to a more pleasant user experience.

\section{CONCLUSIONS}

We evaluated progressive treemaps with regard to their ability to overcome the drawback of traditional treemaps to convey tree topology and node properties. To achieve this, we designed a user study using a common squarified treemap shown in either the traditional way or by progressive refinement and recording the answers of our participant to various objective and subjective questions. We used many different set-ups to allow for broad applicability of the study. We found that progressive treemaps delivered a significantly higher correctness for all considered tasks. The results vary depending on the task. The highest gain was achieved when we asked the participants to the number of hierarchy levels (22.1\%), the lowest, when we asked for the position of the deepest node (5.3\%). Confidence about the given answers (73\%), assistance as well as fun (71\%) provided during task completion were also much higher for the progressive treemap showing that this approach was well-accepted by the participants. However, our data also indicated that the benefits of progressive treemaps decrease for larger and more complex data sets.

This study represents a first attempt to evaluate progressive data displays. In future research, we plan to extend our evaluation of progressive treemaps to include more tasks, interactivity, and geometry-of-interest refinement in order to underpin the findings obtained. The effect of refresh time might be another property to consider as this influences the time to view inner nodes and thus promises to lead to a higher gain when properly adjusted. It might also be of interest to test the properties of progressive refinement when applied for some treemap extensions, such as cushion treemaps. We also plan to test other progressive information displays, such as progressive scatter plots. ${ }^{15}$ This evaluation represents a foundation and meaningful starting point for these future investigations, and provides valuable information to show the eligibility of progressive information presentation.

\section{Acknowledgements}

The authors gratefully acknowledge the support of Deutsche Forschungsgemeinschaft (DFG) for funding this research (\#RO3755/1-1). 


\section{REFERENCES}

1. R. Rosenbaum and B. Hamann, "Progressive presentation of large hierarchies using treemaps," in Proceedings of 5th International Symposium on Visual Computing 2009, Nov. 2009.

2. T. Barlow and P. Neville, "A comparison of 2-D visualizations of hierarchies," in Proceedings of the IEEE Symposium on Information Visualization 2001 (INFOVIS'01), p. 131, IEEE Computer Society, 2001.

3. B. Shneiderman, "Tree visualization with tree-maps: 2-d space-filling approach," ACM Trans. Graph. 11(1), pp. 92-99, 1992.

4. M. Bruls, K. Huizing, and J. van Wijk, "Squarified treemaps," Proceedings of the joint Eurographics and IEEE TCVG symposium on visualization, pp. 33-42, 1999.

5. J. J. Van Wijk and H. Van de Wetering, "Cushion treemaps: visualization of hierarchical information," in 1999 IEEE Symposium on Information Visualization, 1999. (Info Vis '99) Proceedings, pp. 73-78, 147, IEEE, 1999.

6. M. Balzer and O. Deussen, "Voronoi treemaps," in Proceedings of the Proceedings of the 2005 IEEE Symposium on Information Visualization, p. 7, IEEE Computer Society, 2005. ACM ID: 1106574.

7. R. Vliegen, J. J. van Wijk, and E. van der Linden, "Visualizing business data with generalized treemaps," IEEE Transactions on Visualization and Computer Graphics 12, p. 789796, Sept. 2006. ACM ID: 1187784.

8. R. O'Donnell, A. Dix, and L. Ball, "Ball exploring the PieTree for representing numerical hierarchical data," in Proceedings of HCI2006, Springer, (London), Sept. 2006.

9. T. Bladh, D. Carr, and M. Kljun, "The effect of animated transitions on user navigation in 3D Tree-Maps," in Ninth International Conference on Information Visualisation (IV'05), pp. 297-305, (London, England), 2005.

10. H. L and J. Fogarty, "Cascaded treemaps: examining the visibility and stability of structure in treemaps," in Proceedings of graphics interface 2008, GI '08, p. 259266, Canadian Information Processing Society, (Windsor, Ontario, Canada), 2008. ACM ID: 1375758.

11. Y. Tu and H. Shen, "Balloon focus: a seamless Multi-Focus+Context method for treemaps," IEEE Transactions on Visualization and Computer Graphics 14(6), pp. 1157-1164, 2008.

12. A. Kobsa, "User experiments with tree visualization systems," in Proceedings of the IEEE Symposium on Information Visualization, pp. 9-16, IEEE Computer Society, 2004.

13. R. Blanch and E. Lecolinet, "Browsing zoomable treemaps: Structure-Aware Multi-Scale navigation techniques," IEEE Transactions on Visualization and Computer Graphics 13(6), pp. 1248-1253, 2007.

14. R. Rosenbaum and H. Schumann, "Progressive refinement - more than a means to overcome limited bandwidth," in Proceedings of Electronic Imaging - Visualization and Data Analysis 2009, Jan. 2009.

15. R. Rosenbaum, A. Gimenez, H. Schumann, and B. Hamann, "A flexible low-complexity device adaptation approach for data presentation," pp. 78680F-78680F-12, (San Francisco Airport, California, USA), 2011.

16. B. Shneiderman, "The eyes have it: A task by data type taxonomy for information visualizations," Proceedings of the IEEE Symposium on Visual Languages, pp. 336-343, 1996.

17. J. Stasko, R. Catrambone, M. Guzdial, and K. McDonald, "An evaluation of space-filling information visualizations for depicting hierarchical structures," International Journal of Human-Computer Studies 53, p. 663694, Nov. 2000. ACM ID: 365438 .

18. C. Plaisant, J. Grosjean, and B. B. Bederson, "SpaceTree: supporting exploration in large node link tree, design evolution and empirical evaluation," in Information Visualization, IEEE Symposium on, 0, p. 57, IEEE Computer Society, (Los Alamitos, CA, USA), 2002.

19. K. Andrews and J. Kasanicka, "A comparative study of four hierarchy browsers using the hierarchical visualisation testing environment (HVTE)," in Proceedings of the 11th International Conference Information Visualization, p. 8186, IEEE Computer Society, 2007. ACM ID: 1271570.

20. H. Song, B. Kim, B. Lee, and J. Seo, "A comparative evaluation on tree visualization methods for hierarchical structures with large fan-outs," in Proceedings of the 28th international conference on Human factors in computing systems, pp. 223-232, ACM, (Atlanta, Georgia, USA), 2010.

21. J. Heer and M. Bostock, "Crowdsourcing graphical perception: using mechanical turk to assess visualization design," in Proceedings of the 28th international conference on Human factors in computing systems, pp. 203212, ACM, (Atlanta, Georgia, USA), 2010. 